CUAD. CONTAB. / BOGOTÁ, COLOMBIA, 15 (38): 501-523 / JULIO-DICIEMBRE 2014 / 501

\title{
La perspectiva de los profesionales de la salud acerca de la información contable en un hospital-empresa en Portugal. Estudio de caso*
}

doi: 10.11144/Javeriana.cc15-38.ppsa

\section{Amélia Cristina Ferreira da Silva}

Profesora del departamento de Contabilidad del Instituto Superior de Contabilidade e Administração del Instituto Politécnico do Porto (IPP). Doctora en contabilidad, Universidad de Vigo, España. Máster en contabilidad y auditoría, Universidad do Minho, Portugal. Licenciada en administración, Universidad Moderna, Portugal. Miembro del Centro de Estudos da População, Economia e Sociedade, CEPESE. Miembro del Centro de Estudos em Ciências Empresariais e Jurídicas, CECEJ.

Correo electrónico: acfs@iscap.ipp.pt

\author{
José Manuel Teixeira Pereira \\ Profesor del departamento de Contabilidad y Fiscalidad \\ de la Escola Superior de Gestão del Instituto Politécni- \\ co do Cávado e do Ave, IPCA. Doctor en contabilidad, \\ Universidad de Vigo, España. Máster en contabilidad y \\ auditoría, Universidad do Minho, Portugal. Licenciado \\ en administración de empresas, Universidad da Beira \\ Interior, Portugal. Miembro del Centro de Investigação em \\ Contabilidade e Fiscalidade, CICF, del IPCA. \\ Correo electrónico: jpereira@ipca.pt
}

\footnotetext{
* Este artículo es producto del proyecto de investigación Markets and Organizations Dynamics, vinculado al grupo de investigación Social and Economic Changes: Organizations and Sustainability, de la línea de investigación Organizations, Development and Wellbeing, de CEPESE, Universidad de Oporto.
} 
Resumen Aunque en el contexto internacional sea muy estudiada, en Portugal la utilización de la información económico-financiera en el hospital público es un tema de investigación reciente. Nuestro trabajo está basado en un estudio de caso y tiene como objetivo aclarar si los profesionales que tienen poder de decisión sobre los recursos dentro del hospital, en particular los médicos y los cargos de jefe, usan la información económico-financiera disponible y cuáles son los factores que condicionan su utilización de esta información. Los resultados indican que la consulta de la información económico-financiera no es una práctica común para la generalidad de los médicos y jefes dentro del hospital, aunque un porcentaje importante de profesionales ya la integró en su rutina. El análisis estadístico confirmó solo dos de nuestras hipótesis. Por consiguiente, creemos que además de las variables explicativas identificadas en nuestro estudio, hay espacio para que futuras investigaciones identifiquen otros factores que explican la utilización de la información contable por parte de los profesionales de la salud.

Palabras clave Servicios de salud; gestión pública

\section{Código JEL M41}

\section{The Perspective of Health Professionals on the Accounting Information in a Company-Hospital in Portugal Case Study}

\footnotetext{
Abstract Although it has been greatly studied in the international context, the use of the economic and financial information in public hospitals is a recent research topic in Portugal. Our work is based on case study and its purpose is to make clear whether the professionals in the hospital that have decision-making power on the resources, particularly doctors and chief positions, use the available economic and financial information, and what are the factors that condition their use of this information. The results show that consulting the economic and
}

financial information is not a common practice for most of the doctors and chiefs in the hospital, although an important percentage of the professionals did make this a part of their routines. The statistical analysis confirmed just two of our hypotheses. Thus, we believe that, besides the explanatory variables identified in our study, there is place for future research to identify other factors that explain the use of the accounting information by health professionals.

Keywords health services; public management

\section{A perspectiva dos profissionais de saúde sobre a informação contabilística num hospital-empresa em Portugal. Estudo de caso}

Resumo Apesar de a nível internacional a investigação sobre a utilização da informação económico- financeira em contexto hospitalar ser abundante, em Portugal é um tema de investigação recente. O objectivo deste trabalho é saber se os profissionais que têm poder de decisão sobre os recursos dentro do hospital, em particular os médicos e outros profissionais de saúde com cargos de chefia, usam a informação económico-financeira disponível e quais os fatores que condicionam a utilização dessa informação. Foi realizado um estudo de caso e os resultados indicam que a consulta da informação económico-financeira não é uma prática comum entre os profissionais de saúde, ainda que uma percentagem importante dos profissionais já integre essa informação na sua rotina. A análise estatística confirmou apenas duas das nossas hipóteses sobre os factores que condicionam a utilização desta informação. Acreditamos que, além das variáveis explicativas identificadas no nosso estudo, há outros factores que explicam a utilização da informação contabilística por parte dos profissionais de saúde e por isso é importante continuar a investigar sobre esta questão.

Palavras-chave Serviços de saúde; gestão pública 


\section{Introducción}

Hay una amplia aceptación de la idea de que la contabilidad — sus prácticas y su papel—solo puede ser entendida como parte de los contextos políticos, económicos, sociales y culturales en los que opera (Chua, 1995; Hopwood, 1984; Kaplan, 1983; Lawrence, Alam, Northcott \& Lowe, 1997). En este sentido, desde la década de 1990, la contabilidad de gestión en los servicios de la salud ha sido objeto de un esfuerzo de investigación continuo de forma que su problemática está bien documentada en el ámbito internacional (Abernethy \& Vagnoni, 2004; Aidemark, 2001; Arnaboldi \& Lapsley, 2004; Brickley, Horn \& Wedig, 2010; Chua \& Preston, 1994; Comerford \& Abernethy, 1999; Grafton, Abernethy \& Lillis, 2011; Kurunmäki, 2004; Lawrence, Alan \& Lowe, 1994; Pettersen, 2001). Se trata de un tema que ha sido muy debatido y que puede ser descrito como un "área problemática, llena de controversia y tensión" (Scarparo, 2004). Inspirado en esos estudios, nuestro trabajo está motivado por la voluntad de explorar la realidad portuguesa en relación con la utilización de la información económico-financiera en el contexto hospitalario, materia en que la investigación está todavía poco avanzada.

A finales de la década de los 70, la nueva gestión pública (Farnham \& Horton, 1996; Hood, 1991, 1995; Minogue, 1998) se impone como una corriente dominante de la reforma de la administración pública de los países desarrollados. La idea clave de la nueva gestión pública es que la aplicación de los principios e instrumentos de gestión privada en la admi- nistración pública promueve su eficiencia. En consecuencia, los sistemas de información de las entidades públicas en las que se introducen modelos de gestión empresarial de la administración pública sufren profundas reestructuraciones.

Sin embargo, por las especificidades inherentes al buen "cuidado de la salud", solo recientemente el sector de la salud se ha visto afectado por las reformas enmarcadas en el modelo de la nueva gestión pública. En Portugal, en particular en el subsector hospitalario, la transformación de treinta y cuatro (34) hospitales públicos del sector público administrativo (SPA) en treinta y un (31) Hospitales S.A., hoy Hospitales EPE - Entidad Pública Empresarial, que representan aproximadamente el 50\% de la red de Hospitales Públicos, constituyó la medida más emblemática de la influencia de la corriente de la nueva gestión pública en la reforma del Servicio Nacional de Salud, SNS. La desburocratización de los procesos de adquisición, la posibilidad de realizar contratos individuales de trabajo y la financiación en función de la producción mediante la figura del contrato-programa, constituyen los puntos esenciales de diferenciación de la gestión de los Hospitales S.A. en relación con la de los Hospitales SPA.

Con la intención de apoyar y supervisar la implementación de la reforma en los Hospitales S.A., se creó la Unidad de Misión. Una de las funciones de este grupo de trabajo fue la reestructuración de los sistemas de información económico-financiera, mediante la introducción del cuadro de mando integral (tableau de bord), la promoción de prácticas de benchmar- 
king entre los Hospitales S.A. y la creación y publicación de un informe de los Hospitales S.A., que presentan diversos indicadores de actividad de los diferentes Hospitales S.A. y se ordenan en un ranking en función del "índice global de eficiencia"', que fue publicado en 2005. Con la transformación de los Hospitales, S.A. en Entidades Públicas Empresariales, en el año 2006, fue abandonado el índice global de eficiencia, aunque se mantienen hasta hoy (2014) las prácticas de benchmarking y la divulgación de los indicadores de desempeño económico y financieros.

Dadas las especificidades de los servicios prestados en el hospital público, la independencia clínica inherente al acto médico y las estructuras organizativas dominantes en la institución hospitalaria, el poder de decisión acerca del consumo y la utilización de los recursos en los hospitales públicos está repartido, esencialmente, entre médicos, cargos de jefatura de las estructuras clínicas (en particular, enfermeros jefes y médicos directores) y el consejo de administración (mayoritaria y a veces, exclusivamente, integrado por profesionales de la salud).

En este contexto, se plantea la siguiente pregunta: ¿los profesionales que tienen poder de decisión sobre la disposición de recursos en el hospital-empresa realmente utilizan la información económico-financiera disponible? Y, en

1 Relación entre la "producción del hospital a coste medio de la red, ajustada por el casemix y el factor de estructura del hospital" y la "producción del hospital a costes reales del hospital". El índice de casemix se mide por el peso relativo de cada grupo relacionado por el diagnóstico, que se publica en la Portaría (internamiento, cirugía de ambulatorio y “adaptación” para consulta externa). caso afirmativo, ¿con qué finalidad la utilizan? Por otra parte, en aquellos casos en que no se hace uso de la información económico-financiera, ¿cuál es el motivo? En definitiva, se trata de saber el grado de uso y los factores que condicionan la utilización de esta información.

La reestructuración de los sistemas de información de los Hospitales S.A. y su respectivo seguimiento fueron concebidos por la Unidad de Misión. En un estudio para evaluar la experiencia obtenida con la creación de los Hospitales S.A. en las dimensiones de eficiencia, equidad, acceso y calidad, Miguel Gouveia, José Luís Alvim, Cristina Neto de Carvalho, José António Meneses-Correia y Manuela Mota-Pinto (2006) destacaban la necesidad de mejorar el sistema interno de información económicofinanciera y promover su integración en los procesos de decisión de los directores clínicos $y$, con base en las evidencias empíricas obtenidas, concluía que, a pesar de la complejidad de las cuestiones financieras con que se enfrentan actualmente los hospitales, se impone la urgencia de mejorar significativamente el grado de formación y experiencia de los funcionarios de los departamentos financieros.

Con el propósito de contribuir al esclarecimiento de estas cuestiones, hemos realizado un estudio de caso en uno de estos hospitales que, por razones de confidencialidad, designamos Hospital de Estudio, HE. En diciembre de 2002, el HE se transformó en sociedad anónima de capital exclusivamente público y, posteriormente, pasó a ser Entidad Pública Empresarial.

A partir de la revisión de la literatura consultada sobre evidencias de experiencias relativas a 
la reestructuración y utilización de los sistemas de información económico-financiera en hospitales de otros países y considerando las particularidades de la reforma reciente llevada a cabo en los hospitales públicos portugueses, en la cual se inserta la empresarialización de los hospitales públicos, procedimos a realizar un análisis de potenciales factores que condicionan la utilización de la información contable por los profesionales que gestionan los recursos en el hospital-empresa portugués. Las cuestiones estudiadas pueden enmarcarse en seis áreas:

1. Nivel de conformidad del sujeto con los principios orientadores de la reforma que afectan directamente el funcionamiento interno del hospital.

2. Apreciación del sujeto frente a los efectos económico-financieros del propio proceso de empresarialización en el HE.

3. Apreciación del sujeto frente a la calidad del sistema de información del HE.

4. Perfil profesional del sujeto, en el que se valora el hecho de que hubiera desempeñado cargos de jefatura.

5. Percepción del sujeto sobre su nivel de conocimiento de los conceptos e indicadores clínicos y económico-financieros utilizados en los documentos contables internos.

6. Finalidades de la consulta y motivos de la no consulta de la información contable.

La utilización de la información económicofinanciera en el hospital-empresa es un tema reciente en contabilidad, en relación con el cual hasta el momento hay escasa investigación, en particular en el contexto portugués. Se trata pues de un trabajo necesariamente exploratorio.

\section{Método}

\subsection{Tipo de estudio}

En los años 80, autores como Anthony G. Hopwood (1984) y Robert Kaplan (1983) reclamaban la necesidad de desarrollar la investigación empírica en el campo de la contabilidad de gestión, a fin de saber cómo funciona la contabilidad de gestión en el entorno en que opera, para intentar captar las ambigüedades y contradicciones de las organizaciones donde trabajan los contables y disminuir el gap existente entre la investigación académica y la práctica de la contabilidad de gestión (Drury \& Tayles, 1995; Edwards \& Emmanuel, 1990). En este sentido, el proclamado potencial de los estudios de campo - en particular, de los estudios de caso- está asociado a la convicción de que "no se puede estudiar la Contabilidad como una actividad técnica y racional que está separada de relaciones sociales más amplias" (Chua, 1986).

Así, el método de estudio de caso (Yin, 1994) se presenta como una alternativa metodológica para la exploración de nuevos fenómenos y es aplicado y defendido por diversos autores como un instrumento que permite explorar nuevas relaciones teóricas y cuestionar las viejas teorías (Ahrens \& Dent, 1998; Keating, 1995). Por otro lado, como sostiene Robert W. Scapens (1994), "los investigadores no deben quedar excesivamente comprometidos en la comparación de las prácticas de contabilidad de gestión con los 'ideales' teóricos. Por el contrario, deben enfocarse más hacia el estudio de la práctica de la contabilidad de gestión per se". Nosotros aceptamos la complejidad, la ambigüedad y la contradicción como parte de la vida diaria y 
reconocemos esas características como "aspectos esenciales e inevitables del proceso de investigación y del entorno natural en que la investigación discurre" (Lawrence, Alam \& Lowe, 1994).

Reconociendo que el sistema de información contable y el papel de la contabilidad en el hospital-empresa portugués son áreas prácticamente carentes de investigación, debido a las profundas alteraciones de que ha sido objeto hemos estado por la realización de un estudio de caso, nuestra elección metodológica se fundamenta en el hecho de que - dada la escasez de estudios anteriores sobre la problemática relacionada con el uso de la información económico-financiera en el hospital-empresa, y atendiendo al complejo proceso de transformación que ha atravesado y atraviesa el sector hospitalario portugués- el estudio de un caso tendría, frente a metodologías alternativas, la ventaja de permitirnos captar toda la complejidad que envuelve las respuestas a nuestras preguntas de partida.

Para la recogida de datos, hemos utilizado la técnica de la entrevista con base en un cuestionario que plantea preguntas cerradas, con una escala Likert de 5, precedido de una entrevista semiestructurada con el presidente del Consejo de Administración, del análisis de diversos documentos contables internos y externos y de diversas reuniones con el responsable de la contabilidad y con el administrador ejecutivo.

La razón de esta elección es que el HE fue uno de los diez hospitales piloto del proyecto de los Hospitales S.A. Los motivos de su inclusión no están totalmente claros, pero hay indicaciones de que se debió a su elevado potencial de mejoría, tanto económica como financiera. Se trata de una unidad hospitalaria regional, que forma parte de la Red hospitalaria desde 1986. Atiende una población de cincuenta mil habitantes y puede ser clasificado como semirrural. Este hospital ofrece las especialidades de medicina interna, cirugía general, pediatría, psiquiatría agudos, psiquiatría crónicos, obstetricia, neonatología y ortopedia; tiene 179 camas, servicio de urgencias básicas y consultas externas.

Durante la entrevista, el presidente del Consejo de Administración comentó frecuentemente las dificultades que entraña la gestión de los recursos humanos en el contexto hospitalario:

La gran diferencia frente a la gestión de una empresa, una fábrica por ejemplo, es que mientras que el gestor de la fábrica tiene puntos de producción predefinidos con una jerarquía muy rígida, en el hospital eso no sucede. El gran problema de los hospitales es fundamentalmente de relaciones humanas [...] el 80\% de los Recursos Humanos son cuadros superiores, con intereses distintos, altamente especializados y cualificados. Por tanto, el Consejo de Administración debe tener un conocimiento profundo de esta realidad, desarrollando las relaciones humanas con el otro lado, incentivándolas y motivándolas en aras del crecimiento de producción, etc.

En los documentos internos analizados (informe de gestión y plan de negocios), se hace patente una filosofía de gestión empresarial, en la cual destacamos el uso de la palabra clientes para referirse a los usuarios de los cuidados de salud, lo que en nuestra opinión puede deberse al hecho de que el administrador del HE es un administrador hospitalario de carrera. Además, a lo largo de 
la entrevista, utilizó un conjunto de expresiones, comentarios y afirmaciones que refuerzan esta visión empresarial de la gestión del hospital y la importancia otorgada a la información económico-financiera en el proceso de decisión.

La financiación asignada depende de la cantidad de producción contratada para cada una de las líneas de producción. Esta producción se mide en Grupos Relacionados por el Diagnóstico (GRD) y se ajusta por el índice de casemix (severidad media de las patologías). Así, el precio que el Ministerio de Salud paga a los hospitales depende de la cantidad de producción, del tipo de producción (GRD) y de la complejidad general de los casos tratados en el hospital (casemix). Estos precios son definidos por el Ministerio de la Salud y hay tres tablas diferentes de precios: Grupo I, Grupo II y Grupo III. Estos grupos corresponden a clusters que obedecen al nivel de diferenciación clínica y a la matriz tecnológica del hospital.

El HE presenta un conjunto de factores que presionan a favor de una gestión especialmente crítica de los recursos, lo que nos lleva a pensar que hay una mayor sensibilidad a los principios de racionalidad económica en la toma de decisiones, lo que asociamos con una mayor necesidad de información económico-financiera, es decir, presenta un contexto organizativo favorable a la promoción de su utilización.

\subsection{Cuestiones de estudio}

En cuanto al grado de conformidad del entrevistado con los principios orientadores de la reforma que afectan directamente el funcionamiento interno del hospital, planteamos once (11) hipótesis:
Hipótesis 1: Hay diferencias en el grado de aceptación de la misión empresarial del Hospital Público, en función de si el sujeto utiliza la información económico-financiera o no lo hace.

Hipótesis 2: Hay diferencias en el grado de aceptación de la evaluación externa del Hospital Público, en función de si el sujeto utiliza la información económico-financiera o no lo hace.

Hipótesis 3: Hay diferencias en el grado de aceptación de la publicación de los resultados obtenidos en la evaluación externa del hospital público, en función de si el sujeto utiliza la información económico-financiera o no lo hace. Hipótesis 4: Hay diferencias en la forma en que los profesionales de la salud perciben su responsabilidad en el desempeño económico de los hospitales, en función de si el sujeto utiliza la información económico-financiera o no lo hace.

Hipótesis 5: Hay diferencias en el grado de aceptación del modelo de financiación, en función de la producción dependiendo de si el sujeto utiliza la información económicofinanciera o no lo hace.

Hipótesis 6: Hay diferencias en el grado de aceptación de la atribución de la administración hospitalaria a los gestores profesionales, en función de si el sujeto utiliza la información económico-financiera o no lo hace.

Con respecto a la apreciación del sujeto frente a los efectos económicos y financieros derivados del proceso de empresarialización en el propio HE, se trata de analizar si: 
Hipótesis 7: Hay diferencias en la apreciación de los efectos económico-financieros derivados de la empresarialización en el HE, en función de si el sujeto utiliza la información económico-financiera o no lo hace.

En relación con la apreciación del sujeto frente a la calidad del sistema de información del HE se intentará comprobar si:

Hipótesis 8: Hay diferencias en la apreciación cualitativa del sistema de información, en función de si el sujeto utiliza la información económico-financiera o no lo hace.

En cuanto a la percepción del propio sujeto sobre su nivel de conocimiento de los conceptos e indicadores clínicos y económico-financieros utilizados en los documentos contables, analizamos la siguiente hipótesis:
Hipótesis 9: Hay diferencias en el grado de comprensión de los conceptos e indicadores clínicos, en función de si el sujeto utiliza la información económico-financiera o no lo hace. Hipótesis 10: Hay diferencias en el grado de comprensión de los conceptos e indicadores económico-financieros, en función de si el sujeto utiliza la información económico-financiera o no lo hace.

En relación con el perfil profesional de los sujetos, exploramos la siguiente cuestión:

Hipótesis 11: Hay una asociación entre el ejercicio del cargo de jefe y la utilización de la información económico-financiera.

Por último, nos propusimos identificar la finalidad con que se consulta la información económico-financiera y los motivos para no consultar los documentos contables.

\begin{tabular}{|c|c|c|c|}
\hline Dimensión/afirmaciones & \multicolumn{2}{|r|}{ Codificación } & Interpretación \\
\hline Misión del hospital & $\Sigma / 3=[0-5]$ & \multirow{4}{*}{$\begin{array}{l}\text { 1. Totalmente en } \\
\text { desacuerdo } \\
\text { 2. Parcialmente en } \\
\text { desacuerdo } \\
\text { 3. No está de acuerdo ni en } \\
\text { desacuerdo } \\
\text { 4. Parcialmente de acuerdo } \\
\text { 5. Totalmente de acuerdo }\end{array}$} & \multirow{4}{*}{$\begin{array}{l}\text { Cuanto mayor es el } \\
\text { grado de concordancia } \\
\text { menor es la aceptación } \\
\text { de la misión de cariz } \\
\text { empresarial en el } \\
\text { hospital público. }\end{array}$} \\
\hline $\begin{array}{l}\text { La misión del hospital público es proteger a los más } \\
\text { desfavorecidos. }\end{array}$ & $1,2,3,4,5$ & & \\
\hline $\begin{array}{l}\text { La misión del hospital público es prestar cuidados } \\
\text { de salud diferenciados sin preocupaciones } \\
\text { económicas. }\end{array}$ & $1,2,3,4,5$ & & \\
\hline $\begin{array}{l}\text { El objetivo del lucro es incompatible con la misión } \\
\text { del hospital público. }\end{array}$ & $1,2,3,4,5$ & & \\
\hline Evaluación externa de los hospitales & $\Sigma / 3=[0-5]$ & \multirow{4}{*}{$\begin{array}{l}\text { 1. Totalmente en } \\
\text { desacuerdo } \\
\text { 2. Parcialmente en } \\
\text { desacuerdo } \\
\text { 3. No está de acuerdo ni en } \\
\text { desacuerdo } \\
\text { 4. Parcialmente de acuerdo } \\
\text { 5. Totalmente de acuerdo }\end{array}$} & \multirow{4}{*}{$\begin{array}{l}\text { Cuanto mayor } \\
\text { es el grado de } \\
\text { concordancia, mayor } \\
\text { es la aceptación de la } \\
\text { evaluación externa de } \\
\text { los hospitales. }\end{array}$} \\
\hline $\begin{array}{l}\text { Todos los hospitales públicos deberían ser } \\
\text { evaluados en función de la calidad técnico- } \\
\text { científica de los servicios prestados. }\end{array}$ & $1,2,3,4,5$ & & \\
\hline $\begin{array}{l}\text { Todos los hospitales públicos deberían ser } \\
\text { evaluados en función del grado de satisfacción de } \\
\text { los usuarios. }\end{array}$ & $1,2,3,4,5$ & & \\
\hline $\begin{array}{l}\text { Todos los hospitales públicos deberían ser } \\
\text { evaluados en función de la gestión de los recursos } \\
\text { económicos y financieros. }\end{array}$ & $1,2,3,4,5$ & & \\
\hline
\end{tabular}


LA INFORMACIÓN CONTABLE EN UN HOSPITAL / A. DA SILVA, J. PEREIRA / $\mathbf{5 0 9}$

\begin{tabular}{|c|c|c|c|}
\hline Dimensión/afirmaciones & \multicolumn{2}{|r|}{ Codificación } & Interpretación \\
\hline $\begin{array}{l}\text { Publicación de los resultados de la evaluación } \\
\text { externa de los hospitales }\end{array}$ & $\Sigma / 2=[0-5]$ & \multirow{3}{*}{$\begin{array}{l}\text { 1. Totalmente en } \\
\text { desacuerdo } \\
\text { 2. Parcialmente en } \\
\text { desacuerdo } \\
\text { 3. No está de acuerdo ni en } \\
\text { desacuerdo } \\
\text { 4. Parcialmente de acuerdo } \\
\text { 5. Totalmente de acuerdo }\end{array}$} & \multirow{3}{*}{$\begin{array}{l}\text { Cuanto mayor es el } \\
\text { grado de concordancia, } \\
\text { mayor es la aceptación } \\
\text { de la divulgación de } \\
\text { los resultados de la } \\
\text { evaluación externa de } \\
\text { los hospitales. }\end{array}$} \\
\hline $\begin{array}{l}\text { Los resultados de la evaluación externa de los } \\
\text { hospitales públicos deberían ser divulgados entre } \\
\text { los ciudadanos. }\end{array}$ & $1,2,3,4,5$ & & \\
\hline $\begin{array}{l}\text { Debería ser creado y publicado un ranking de la } \\
\text { actividad global de todos los hospitales públicos. }\end{array}$ & $1,2,3,4,5$ & & \\
\hline $\begin{array}{l}\text { Papel y responsabilidad de los profesionales de la } \\
\text { salud en el desempeño económico de los hospitales }\end{array}$ & $\Sigma / 4=[0-5]$ & \multirow{5}{*}{$\begin{array}{l}\text { 1. Totalmente en } \\
\text { desacuerdo } \\
\text { 2. Parcialmente en } \\
\text { desacuerdo } \\
\text { 3. No está de acuerdo ni en } \\
\text { desacuerdo } \\
\text { 4. Parcialmente de acuerdo } \\
\text { 5. Totalmente de acuerdo }\end{array}$} & \multirow{5}{*}{$\begin{array}{l}\text { Cuanto mayor } \\
\text { es el grado de } \\
\text { concordancia, mayor } \\
\text { es la aceptación de la } \\
\text { importancia del papel } \\
\text { de los profesionales de } \\
\text { salud en la actividad } \\
\text { económica de los } \\
\text { hospitales. }\end{array}$} \\
\hline $\begin{array}{l}\text { Todos los profesionales de la salud deberían poseer } \\
\text { formación básica en el área de gestión. }\end{array}$ & $1,2,3,4,5$ & & \\
\hline $\begin{array}{l}\text { Debería suministrarse información clara y } \\
\text { objetiva a los profesionales de la salud sobre las } \\
\text { consecuencias económico-financieras de sus } \\
\text { decisiones clínicas. }\end{array}$ & $1,2,3,4,5$ & & \\
\hline $\begin{array}{l}\text { Si los profesionales de la salud tuviesen cono- } \\
\text { cimiento de los efectos económico-financieros } \\
\text { concretos de sus decisiones clínicas, ciertamente } \\
\text { cambiarían su comportamiento en el sentido de una } \\
\text { gestión más crítica de los recursos sin que se pusie- } \\
\text { se en duda la calidad de los servicios prestados. }\end{array}$ & $1,2,3,4,5$ & & \\
\hline $\begin{array}{l}\text { Deberían ser concedidos incentivos a los profesio- } \\
\text { nales de la salud que demostrasen mayor preocupa- } \\
\text { ción con las consecuencias económico-financieras } \\
\text { de sus decisiones, sin que se pusiese en duda la cali- } \\
\text { dad de los servicios prestados }\end{array}$ & $1,2,3,4,5$ & & \\
\hline $\begin{array}{l}\text { Financiamiento del hospital en función de la } \\
\text { producción }\end{array}$ & $\Sigma / 3=[0-5]$ & \multirow{4}{*}{$\begin{array}{l}\text { 1. Totalmente en } \\
\text { desacuerdo } \\
\text { 2. Parcialmente en } \\
\text { desacuerdo } \\
\text { 3. No está de acuerdo ni en } \\
\text { desacuerdo } \\
\text { 4. Parcialmente de acuerdo } \\
\text { 5. Totalmente de acuerdo }\end{array}$} & \multirow{4}{*}{$\begin{array}{l}\text { Cuanto mayor es el } \\
\text { grado de concordancia, } \\
\text { mayor es la aceptación } \\
\text { de financiación } \\
\text { en función de la } \\
\text { producción. }\end{array}$} \\
\hline $\begin{array}{l}\text { Los hospitales públicos deben estar financiados en } \\
\text { función de la producción. }\end{array}$ & $1,2,3,4,5$ & & \\
\hline $\begin{array}{l}\text { Los servicios clínicos de los hospitales públicos } \\
\text { deben estar financiados en función de la } \\
\text { producción. }\end{array}$ & $1,2,3,4,5$ & & \\
\hline $\begin{array}{l}\text { Los profesionales de salud de los hospitales } \\
\text { públicos deberían ser remunerados en función de } \\
\text { la producción. }\end{array}$ & $1,2,3,4,5$ & & \\
\hline $\begin{array}{l}\text { Administración hospitalaria por gestores } \\
\text { profesionales (con formación en gestión y sin } \\
\text { formación en el área de la salud) }\end{array}$ & $\Sigma / 5=[0-5]$ & \multirow{4}{*}{$\begin{array}{l}\text { 1. Totalmente en } \\
\text { desacuerdo } \\
\text { 2. Parcialmente en } \\
\text { desacuerdo } \\
\text { 3. No está de acuerdo ni en } \\
\text { desacuerdo } \\
\text { 4. Parcialmente de acuerdo } \\
\text { 5. Totalmente de acuerdo }\end{array}$} & \multirow{4}{*}{$\begin{array}{l}\text { Cuanto mayor es el } \\
\text { grado de concordancia, } \\
\text { menor es la aceptación } \\
\text { de la administración } \\
\text { de los hospitales por } \\
\text { gestores profesionales. }\end{array}$} \\
\hline $\begin{array}{l}\text { Si la administración de los hospitales públicos fuera } \\
\text { confiada a gestores profesionales, la autonomía } \\
\text { clínica de los profesionales de la salud podría ser } \\
\text { puesta en duda. }\end{array}$ & $1,2,3,4,5$ & & \\
\hline $\begin{array}{l}\text { La gestión hospitalaria debería estar totalmente } \\
\text { confiada a los profesionales de la salud. }\end{array}$ & $1,2,3,4,5$ & & \\
\hline $\begin{array}{l}\text { Los gestores profesionales tienen principios éticos } \\
\text { totalmente diferentes a los de los profesionales de } \\
\text { la salud. }\end{array}$ & $1,2,3,4,5$ & & \\
\hline
\end{tabular}




\begin{tabular}{|c|c|c|c|}
\hline Dimensión/afirmaciones & \multicolumn{2}{|r|}{ Codificación } & Interpretación \\
\hline $\begin{array}{l}\text { La actividad de los profesionales de la salud no } \\
\text { puede ser evaluada por los gestores. }\end{array}$ & $1,2,3,4,5$ & & \\
\hline $\begin{array}{l}\text { Los gestores tienen demasiado poder dentro de los } \\
\text { hospitales públicos. }\end{array}$ & $1,2,3,4,5$ & & \\
\hline $\begin{array}{l}\text { Efectos económico-financieros de la } \\
\text { empresarialización en el HE }\end{array}$ & $\Sigma / 4=[0-5]$ & $\begin{array}{l}\text { 1. Totalmente en } \\
\text { desacuerdo }\end{array}$ & $\begin{array}{l}\text { Cuanto mayor } \\
\text { es el grado de }\end{array}$ \\
\hline $\begin{array}{l}\text { La transformación del HE en empresa tiene efectos } \\
\text { económico-financieros positivos. }\end{array}$ & $1,2,3,4,5$ & $\begin{array}{l}\text { 2. Parcialmente en } \\
\text { desacuerdo }\end{array}$ & $\begin{array}{l}\text { concordancia, menor } \\
\text { es la apreciación sobre }\end{array}$ \\
\hline $\begin{array}{l}\text { Con la transformación del HE en empresa se notó } \\
\text { una mayor preocupación por la lucha contra el } \\
\text { despilfarro. }\end{array}$ & $1,2,3,4,5$ & $\begin{array}{l}\text { 3. No está de acuerdo ni en } \\
\text { desacuerdo } \\
\text { 4. Parcialmente de acuerdo }\end{array}$ & $\begin{array}{l}\text { los efectos económico- } \\
\text { financieros de la } \\
\text { empresarialización en }\end{array}$ \\
\hline $\begin{array}{l}\text { El HE es un buen ejemplo de cómo es posible } \\
\text { mejorar la actividad económico-financiera sin } \\
\text { poner en duda la calidad de los servicios prestados. }\end{array}$ & $1,2,3,4,5$ & 5. Totalmente de acuerdo & el HE. \\
\hline $\begin{array}{l}\text { El HE es un buen ejemplo de cómo es posible mejorar } \\
\text { la actividad económico-financiera sin poner en duda } \\
\text { las condiciones de trabajo de los profesionales de } \\
\text { salud. }\end{array}$ & $1,2,3,4,5$ & & \\
\hline Apreciación cualitativa del sistema de información & $\Sigma / 7=[0-5]$ & 1. Totalmente en & Cuanto mayor \\
\hline $\begin{array}{l}\text { La información disponible no es relevante para el } \\
\text { ejercicio de mis funciones. }\end{array}$ & $1,2,3,4,5$ & $\begin{array}{l}\text { desacuerdo } \\
\text { 2. Parcialmente en }\end{array}$ & $\begin{array}{l}\text { es el grado de } \\
\text { concordancia, menor }\end{array}$ \\
\hline $\begin{array}{l}\text { La información que necesito para desempeñar } \\
\text { mejor mis funciones no está disponible en tiempo } \\
\text { útil. }\end{array}$ & $1,2,3,4,5$ & $\begin{array}{l}\text { desacuerdo } \\
\text { 3. No está de acuerdo ni en } \\
\text { desacuerdo }\end{array}$ & $\begin{array}{l}\text { es la apreciación } \\
\text { sobre el sistema de } \\
\text { información del HE. }\end{array}$ \\
\hline $\begin{array}{l}\text { No confío en la información disponible, porque los } \\
\text { datos no son fiables. }\end{array}$ & $1,2,3,4,5$ & 5. Totalmente de acuerdo & \\
\hline $\begin{array}{l}\text { La información disponible es de difícil } \\
\text { interpretación. }\end{array}$ & $1,2,3,4,5$ & & \\
\hline $\begin{array}{l}\text { El sistema de información es insuficiente para la } \\
\text { actuación clínica. }\end{array}$ & $1,2,3,4,5$ & & \\
\hline $\begin{array}{l}\text { El sistema de información es insuficiente para la } \\
\text { actuación económico-financiera. }\end{array}$ & $1,2,3,4,5$ & & \\
\hline $\begin{array}{l}\text { El sistema de información fue concebido } \\
\text { sin considerar que tiene que responder a las } \\
\text { necesidades de información de los profesionales de } \\
\text { la salud. }\end{array}$ & $1,2,3,4,5$ & & \\
\hline
\end{tabular}

Tabla 1. Variables y su respectivo sistema de codificación

Fuente: elaboración propia

\subsection{Procedimiento}

Para el análisis preliminar se recogieron diversos documentos: balance, cuenta de resultados, anexo al balance y a la cuenta de resultados, informe de gestión, el Contrato-programa, Plan de negocios y la Memoria de actividades de los
Hospitales S.A. La recogida y análisis de estos datos estuvo acompañada de diversas reuniones informales con el responsable del área financiera y con uno de los administradores ejecutivos. Estas reuniones proporcionaron una visión global de la estructura organizativa del HE, de los 
procesos de cambio en curso y, en particular, de la reestructuración del sistema de información económica y financiera. Con base en la información recogida, se realizó un análisis exhaustivo de los diferentes documentos contables producidos internamente y de los respectivos destinatarios directos.

Después tuvo lugar la entrevista con el presidente del Consejo de Administración. Esta entrevista era esencialmente exploratoria, por lo que apenas se trataron temas y cuestiones directivas. La entrevista fue grabada integralmente y posteriormente enviada por escrito. Para explorar eventuales hipótesis de estudio y contextualizar el análisis de los resultados, realizamos un análisis cualitativo de su contenido.

Tras elaborar una primera versión completa del cuestionario, lo sometimos a la apreciación del presidente del Consejo de Administración del HE y del adjunto del encargado de misión (integrante del Ministerio de la Salud) y, simultáneamente, pusimos a prueba el cuestionario con seis (6) sujetos - un gestor hospitalario y cinco médicos-. De esta fase de prueba resultó la anulación de una de las preguntas. La causa de la anulación fue la baja tasa de respuestas a esta pregunta en particular, en especial por parte de los médicos, hecho que atribuimos a una excesiva especificidad de la pregunta para ese grupo en cuestión, ya que se pedía al sujeto que valorase, utilizando una escala Likert, los documentos consultados con parámetros como periodicidad, formato, detalle, fiabilidad y utilidad. Así mismo, durante todo el proceso nos mantuvimos atentos a todas las señales de rechazo de respuestas motivadas por la posibilidad de que el cuestionario fuese excesivamente largo.
Durante la recopilación de datos, se realizaron aproximadamente doce visitas al hospital con una duración media de cuatro horas. Como principales obstáculos en la obtención de información, podemos señalar la escasa disponibilidad de tiempo por parte de los entrevistados, su preocupación por la confidencialidad de sus respuestas y la poca receptividad hacia este tipo de colaboración.

\section{Resultados}

La muestra está constituida por cuarenta y dos (42) sujetos que desarrollan actualmente su actividad profesional en el HE, descrita en la tabla siguiente:

\begin{tabular}{lc}
\hline \multicolumn{1}{c}{ Sexo } & (\%) \\
\hline Femenino & 52,4 \\
\hline Masculino & 47,6 \\
\hline \multicolumn{1}{c}{ Edad } & (años) \\
\hline Media & 46 \\
\hline Desviación estándar & 8 \\
\hline Media & (añodad \\
\hline Desviación estándar & 13 \\
\hline & 11 \\
\hline Vínculo laboral definitivo & $\mathbf{( \% )}$ \\
\hline Contrato laboral individual & 88,1 \\
\hline \multicolumn{1}{c}{ Funciones } & 11,9 \\
\hline Director de servicio clínico & $\mathbf{( \% )}$ \\
\hline Médico & 16,7 \\
\hline Enfermero-jefe & 47,6 \\
\hline Consejo de administración & 28,6 \\
\hline Tuve formación en gestión & 7,1 \\
\hline Sí & $\mathbf{( \% )}$ \\
\hline No & 37,1 \\
\hline
\end{tabular}

Tabla 2. Caracterización de la muestra Fuente: elaboración propia 
Veintisiete (27) sujetos afirmaron ejercer, o haber ejercido, funciones de jefatura en el HE o en otro establecimiento de salud (el 65,9\% de las respuestas válidas y el 64,3\% del total de la muestra) y catorce (14) sujetos nunca tuvieron ninguna función de jefatura en establecimientos de salud (el 34,1\% de las respuestas válidas y el 33,3\% del total de la muestra). Un sujeto no quiso responder esta pregunta.

Para el tratamiento estadístico de los datos, recurrimos al programa SPSS. Hemos aplicado el test de Mann-Whitney (tabla 3) para comparar dos muestras con variables medidas en escala ordinal y la prueba de chi-cuadrado para calcular diferencias de proporciones.

\begin{tabular}{|c|c|c|c|c|}
\hline Variables & Grupo & $\mathbf{Z}$ & $\mathbf{U}$ & $\mathbf{P}$ \\
\hline \multirow{2}{*}{$\begin{array}{l}\text { Aceptación de la misión de cariz empresarial del hospital } \\
\text { público }\end{array}$} & No usuarios &,- 688 & 124,500 & ,518 (n.s.) \\
\hline & Usuarios & & & \\
\hline \multirow[t]{2}{*}{ Aceptación de la evolución externa del hospital público } & No usuarios &,- 345 & 171,000 & ,769 (n.s.) \\
\hline & Usuarios & & & \\
\hline \multirow{2}{*}{$\begin{array}{l}\text { Aceptación de la publicación de los resultados de la } \\
\text { evaluación externa }\end{array}$} & No usuarios & $-2,542$ & 89,000 &, $016^{*}$ \\
\hline & Usuarios & & & \\
\hline \multirow{2}{*}{$\begin{array}{l}\text { Reconocimiento del papel y responsabilidad de los } \\
\text { profesionales de salud en el desempeño económico de los } \\
\text { hospitales }\end{array}$} & No usuarios & $-1,727$ & 115,500 & ,112 (n.s.) \\
\hline & Usuarios & & & \\
\hline \multirow{2}{*}{$\begin{array}{l}\text { Aceptación del financiamiento del hospital en función de la } \\
\text { producción }\end{array}$} & No usuarios & $-2,507$ & 82,000 & ,018* \\
\hline & Usuarios & & & \\
\hline \multirow{2}{*}{$\begin{array}{l}\text { Aceptación de la administración hospitalario por los gestores } \\
\text { profesionales }\end{array}$} & No usuarios &,- 957 & 127,500 & ,369 (n.s.) \\
\hline & Usuarios & & & \\
\hline \multirow{2}{*}{$\begin{array}{l}\text { Apreciación de los efectos económico-financieros de la } \\
\text { empresarialización en el HE }\end{array}$} & No usuarios & $-1,798$ & 112,000 & ,093 (n.s.) \\
\hline & Usuarios & & & \\
\hline \multirow[t]{2}{*}{ Apreciación cualitativa del sistema de información del HE } & No usuarios &,- 160 & 139,500 & ,882 (n.s.) \\
\hline & Usuarios & & & \\
\hline \multirow[t]{2}{*}{ Nivel de comprensión de los conceptos e indicadores clínicos } & No usuarios & $-1,095$ & 117,500 & ,296 (n.s.) \\
\hline & Usuarios & & & \\
\hline \multirow{2}{*}{$\begin{array}{l}\text { Nivel de comprensión de los conceptos e indicadores } \\
\text { económico-financieros }\end{array}$} & No usuarios & $-1,752$ & 112,000 & ,093 (n.s.) \\
\hline & Usuarios & & & \\
\hline
\end{tabular}

*p $<0,05$

Tabla 3. Resultados del test de Mann-Whitney

Fuente: elaboración propia 
Con base en los datos del test de Mann-Whitney se puede concluir que solo se aprecian diferencias estadísticamente significativas entre los dos grupos con relación a la aceptación de la publicación de los resultados de la evaluación externa $(\mathrm{Z}=-2,542 ; \mathrm{U}=89,000 ; \mathrm{P}=, 016(\mathrm{p}<.05))$ y con relación a la aceptación del modelo de financiación del hospital en función de la producción $(\mathrm{Z}=-2,507 ; \mathrm{U}=82,000 ; \mathrm{P}=, 018(\mathrm{p}<.05))$. $\mathrm{Al}$ observar atentamente los resultados, se constata que el grupo de sujetos que utiliza la información económico-financiera es el que presenta resultados más concordantes con estas variables.
En la muestra se verifica esta misma tendencia de concordancia con relación a las otras variables, pero no confirmada por el test de Mann-Whitney.

A fin de determinar la asociación entre el cargo de jefatura y el uso de información contable, procedemos al cálculo del test chi-cuadrado. Los datos recogidos no revelan la existencia de una asociación estadísticamente significativa entre las variables $\left(\chi^{2}(1)=2,434 ; \mathrm{P}=0,119\right)$.

Por último, se presentan los resultados obtenidos acerca de la finalidad de los sujetos al consultar la información económico-financiera y los motivos para no consultar los documentos contables.

\begin{tabular}{|c|c|c|c|c|c|c|c|c|c|c|}
\hline \multirow{2}{*}{$\begin{array}{c}\text { Niveles de } \\
\text { consulta } \\
\text { Documentos } \\
\text { consultados }\end{array}$} & \multicolumn{2}{|c|}{$\begin{array}{l}\text { Nunca } \\
\text { consultó }\end{array}$} & \multicolumn{2}{|c|}{$\begin{array}{c}\text { Raramente } \\
\text { consultó }\end{array}$} & \multicolumn{2}{|c|}{$\begin{array}{l}\text { Consultó } \\
\text { regularmente }\end{array}$} & \multicolumn{2}{|c|}{$\begin{array}{c}\text { Consultó } \\
\text { frecuentemente }\end{array}$} & \multicolumn{2}{|c|}{$\begin{array}{l}\text { Consultó muy } \\
\text { frecuentemente }\end{array}$} \\
\hline & $\mathbf{N}$ & (\%) & $\mathbf{N}$ & (\%) & $\mathbf{N}$ & (\%) & $\mathbf{N}$ & (\%) & $\mathbf{N}$ & (\%) \\
\hline Informe de & & & & & & & & & & \\
\hline gestión HE & 18 & 45,0 & 11 & 27,5 & 6 & 15,0 & 4 & 10,0 & 1 & 2,50 \\
\hline Presupuesto del & & & & & & & & & & \\
\hline $\mathrm{HE}$ & 24 & 60,0 & 8 & 20,0 & 5 & 12,5 & 2 & 5,0 & 1 & 2,50 \\
\hline $\begin{array}{l}\text { Presupuesto del } \\
\text { servicio donde } \\
\text { trabaja }\end{array}$ & 22 & 56,4 & 5 & 12,8 & 6 & 15,4 & 4 & 10,3 & 2 & 5,10 \\
\hline $\begin{array}{l}\text { Plan de negocios } \\
\text { del HE }\end{array}$ & 31 & 77,5 & 3 & 7,5 & 3 & 7,5 & 1 & 2,5 & 2 & 5,00 \\
\hline $\begin{array}{l}\text { Contrato- } \\
\text { programa del HE }\end{array}$ & 31 & 77,5 & 3 & 7,5 & 3 & 7,5 & 1 & 2,5 & 2 & 5,00 \\
\hline $\begin{array}{l}\text { Cuadro de mando } \\
\text { integral del HE }\end{array}$ & 16 & 40,0 & 6 & 15,0 & 6 & 15,0 & 8 & 20,0 & 4 & 10,00 \\
\hline $\begin{array}{l}\text { Memoria de } \\
\text { actividades } \\
\text { Hospitales S.A. }\end{array}$ & 7 & 17,9 & 12 & 30,8 & 9 & 23,1 & 8 & 20,5 & 3 & 7,10 \\
\hline
\end{tabular}

Tabla 4. Presentación de los resultados referentes a la frecuencia de consulta de los documentos contables Fuente: elaboración propia

$\mathrm{Al}$ revisar los datos del cuadro anterior, los documentos más referenciados por los sujetos como nunca consultados son el plan de negocios del HE (77,50\%) y el contrato entre el HE y el Ministerio de la Salud (77,50\%). Por otro lado, entre los documentos consultados frecuentemente o muy frecuentemente constan como más citados por los sujetos la Memoria de actividades de los Hospitales S.A. (27,60\%) y el cuadro de mando integral del HE (30\%). Además, 
la Memoria de actividades de los Hospitales S.A. es consultada regularmente por 23,10\% de los sujetos. En global, los resultados indican que, en general, los documentos contables nunca son consultados (valores porcentuales iguales superiores a $40 \%)$.

\begin{tabular}{|c|c|c|c|c|c|c|c|c|c|c|}
\hline \multirow[t]{2}{*}{$\begin{array}{l}\text { Finalidad de la } \\
\text { consulta }\end{array}$} & \multicolumn{2}{|c|}{$\begin{array}{l}\text { Tomar una } \\
\text { decisión en } \\
\text { el ámbito } \\
\text { de mis } \\
\text { funciones }\end{array}$} & \multicolumn{2}{|c|}{$\begin{array}{c}\text { Evaluar la } \\
\text { situación } \\
\text { económica } \\
\text { y financiera } \\
\text { del hospital } \\
\text { como } \\
\text { trabajador }\end{array}$} & \multicolumn{2}{|c|}{$\begin{array}{c}\text { Evaluar la } \\
\text { actividad de la } \\
\text { administración } \\
\text { del hospital }\end{array}$} & \multicolumn{2}{|c|}{$\begin{array}{c}\text { Simple } \\
\text { curiosidad }\end{array}$} & \multicolumn{2}{|c|}{ Otro } \\
\hline & $\mathbf{N}$ & (\%) & $\mathbf{N}$ & $(\%)$ & $\mathbf{N}$ & $(\%)$ & $\mathbf{N}$ & (\%) & $\mathbf{N}$ & (\%) \\
\hline Informe de gestión HE & 8 & 42,1 & 5 & 23,5 & 4 & 18,2 & 2 & 4,8 & --- & --- \\
\hline Presupuesto del HE & 4 & 25,0 & 7 & 43,8 & 2 & 4,8 & 3 & 7,1 & --- & --- \\
\hline $\begin{array}{l}\text { Presupuesto del servicio } \\
\text { donde trabaja }\end{array}$ & 10 & 58,8 & 4 & 23,5 & 2 & 11,8 & 1 & 5,9 & --- & --- \\
\hline Plan de negocios del HE & 3 & 27,3 & 2 & 18,2 & 2 & 18,2 & 4 & 36,4 & -- & -- \\
\hline $\begin{array}{l}\text { Contrato-programa del } \\
\mathrm{HE}\end{array}$ & 3 & 30,0 & 2 & 20,0 & 2 & 20,0 & 3 & 30,0 & --- & --- \\
\hline $\begin{array}{l}\text { Cuadro de mando integral } \\
\text { del HE }\end{array}$ & 4 & 19,0 & 5 & 23,8 & 5 & 21,4 & 6 & 28,7 & 1 & 4,80 \\
\hline $\begin{array}{l}\text { Memoria de actividades } \\
\text { Hospitales S.A. }\end{array}$ & 11 & 39,3 & 6 & 21,4 & 5 & 16,7 & 5 & 17,9 & 1 & 3,6 \\
\hline
\end{tabular}

Tabla 5. Presentación de los resultados relativos a los fines que impulsan a los sujetos a consultar los documentos contables Fuente: elaboración propia

A partir de los datos expuestos en la tabla 5, se comprueba que la Memoria de actividades de los Hospitales S.A. (39,30\%) y el presupuesto del servicio donde trabaja $(58,80 \%)$ son los documentos más consultados por los sujetos de la muestra cuando tratan de tomar una decisión en el ámbito de sus funciones. Con el objetivo de evaluar la situación económica y financiera del hospital como trabajador, los sujetos señalan que consultan con más frecuencia el presupuesto del HE (43,80\%). Para evaluar la actividad de la administración del hospital, los entrevistados en este estudio consultan con más frecuencia el cuadro de mando integral del HE (20,40\%), la Memoria de actividades de los Hospitales S.A. (16,70\%) y el informe de ges- tión del HE (18,20\%). Los sujetos mencionan que los documentos que con más frecuencia consultan por simple curiosidad son el cuadro de mando integral $(28,65 \%)$ y la Memoria de actividades de los Hospitales S.A. (17,90\%).

La principal razón indicada por los sujetos como motivo para no consultar los documentos contables es el hecho de que estos no están disponibles (valores porcentuales iguales o superiores a 50\% en todos los ítems). El motivo menos valorado por los sujetos para no consultar la documentación es su no comprensión (valores inferiores a 12,50\% en todos los ítems). Por último, 5 sujetos de la muestra señalan la falta de interés como la causa para no consultar los documentos contables. 


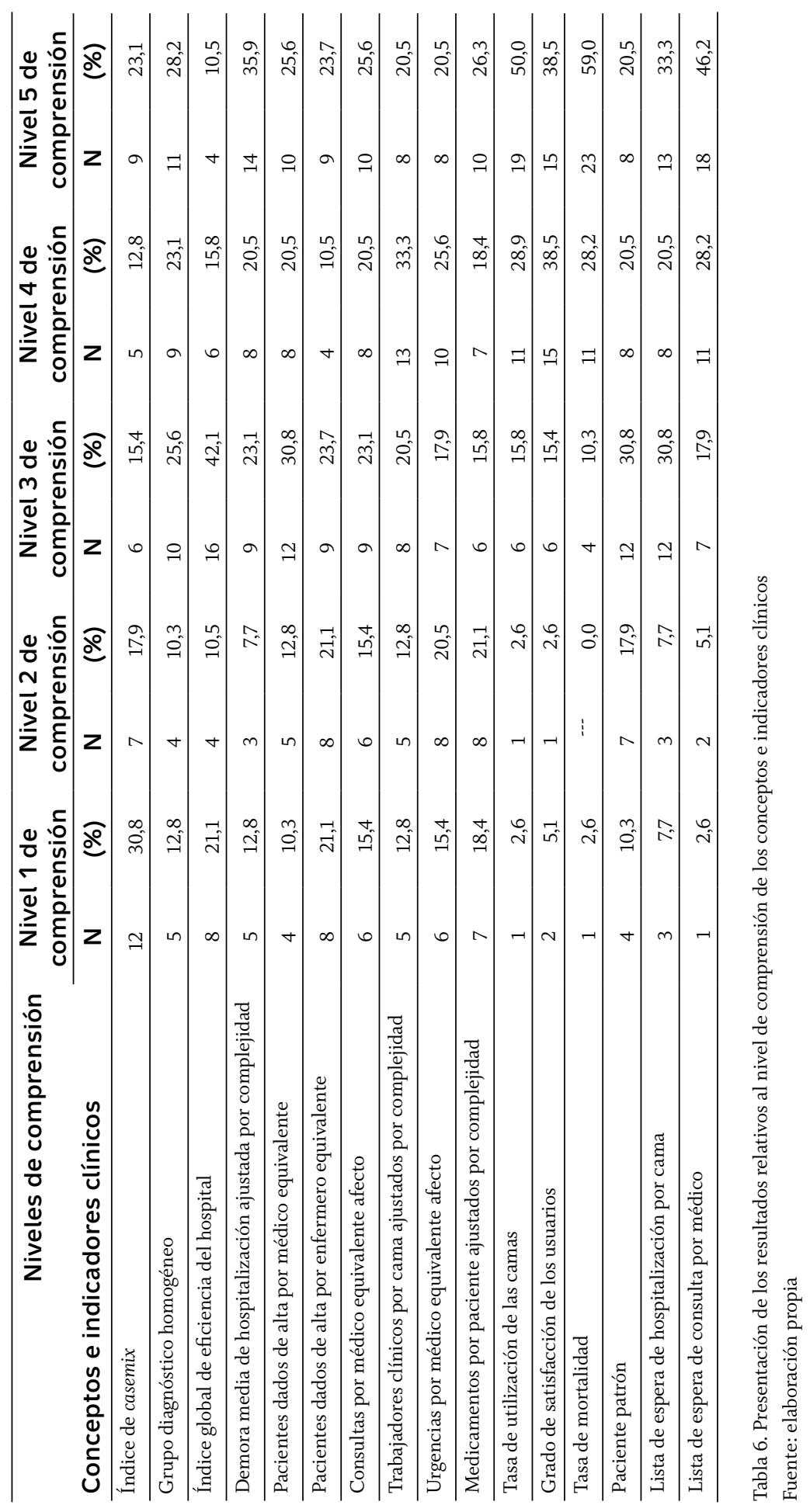


La lectura de los datos recogidos en la tabla 6 nos revela que los sujetos señalan que el índice de casemix es el indicador que más dificultades de comprensión ofrece (30,8\%), seguido del índice global de eficiencia hospitalaria (21,1\%). El indicador clínico que ofrece niveles más elevados de comprensión (nivel 5) es la tasa de mortalidad (59\%).

Con base en los datos de la tabla 7, el indicador benchmarking es el que ofrece más dificultades de comprensión (nivel 1) (55,3\%). Entre 1 y 7 sujetos de la muestra expresan niveles de dificultad bajos (nivel 2) en todos los indicadores económico-financieros considerados. En último lugar, los indicadores económicofinancieros que ofrecen niveles más elevados de comprensión (nivel 5) son ingresos y gastos, y activos y pasivos (cada uno de los ítems con $42,1 \%)$.

\section{Discusión}

El estudio de los factores que condicionan la utilización de la información económico-financiera en el hospital es, sin duda, una cuestión compleja que sobrepasa largamente la exploración de los aspectos técnicos. Este tema tiene que ser enmarcado en una problemática más amplia: la del control del crecimiento del gasto público de salud en un contexto de escasez de recursos, que no admite visiones simplistas y reductoras del tipo: "los médicos son una clase profesional centrada en sí misma y completamente alejada de las consecuencias económicas de sus actos" o "los gestores tienen una visión economicista de la salud y de la vida humana, quieren imponerse a los médicos e invadir su autonomía profesional". En su base, se trata un área donde se mezclan aspectos económicos con cuestiones fundamentales para la vida humana. Es por tanto un área que merece el esfuerzo de un análisis serio y carente de prejuicios.

En particular, a partir de los años, el ritmo de crecimiento del gasto sanitario soportado por el Estado ha creado una gran presión sobre la clase política, en la mayoría de los países de la Unión Europea. Las restricciones presupuestarias llevaron a una reflexión sobre principios, prioridades y organización de los sistemas de salud. Así, del predominio de las políticas sanitarias centradas en la conquista de la equidad y la universalidad en el acceso a los cuidados de salud se pasó a la aplicación de políticas sanitarias centradas en la garantía de la calidad de los cuidados prestados y en la eficiencia económica de las instituciones prestadoras de estos servicios.

En el nivel macro, las soluciones experimentadas han tendido a introducir mecanismos de mercado en el sector de la salud, como la separación entre el papel de prestador y el financiador, la primacía de los modelos de financiación basados en la producción sobre los modelos basados en el gasto, la introducción de la libertad de elección del usuario o el refuerzo de los mecanismos de regulación. En el nivel micro, la adopción de modelos de gestión privada en las instituciones públicas que prestan cuidados de salud es la cara más visible de la influencia de la nueva gestión pública en el sector de la salud.

Uno de estos desafíos es la introducción de los principios de racionalidad económica en el 


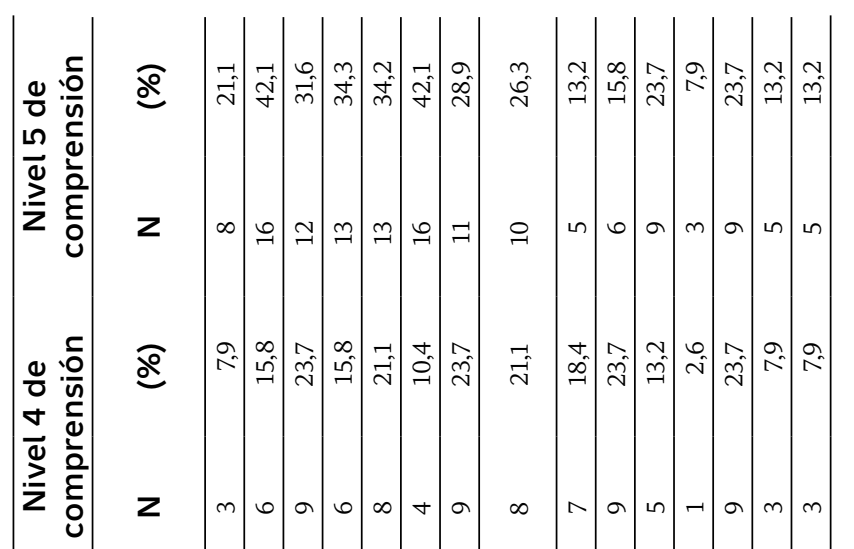

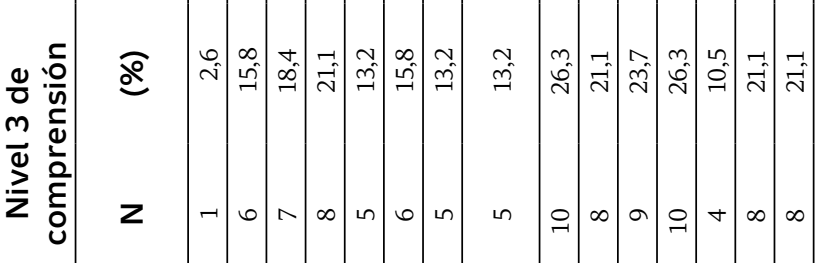

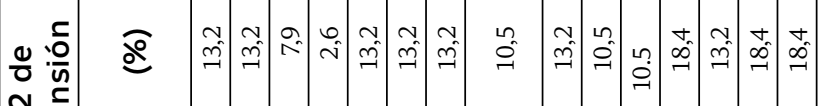

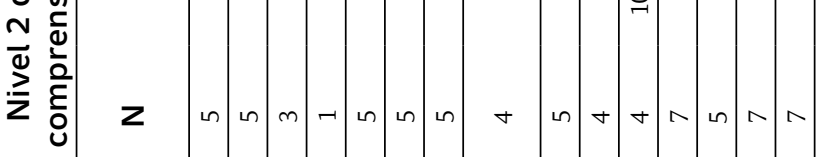

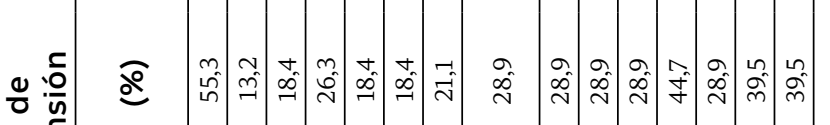

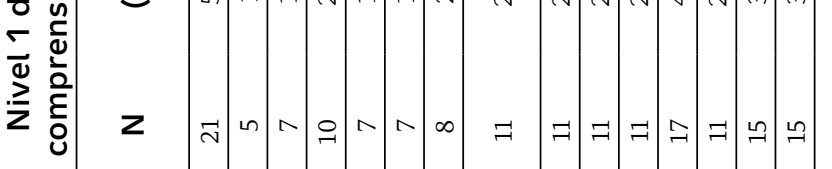

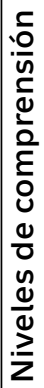

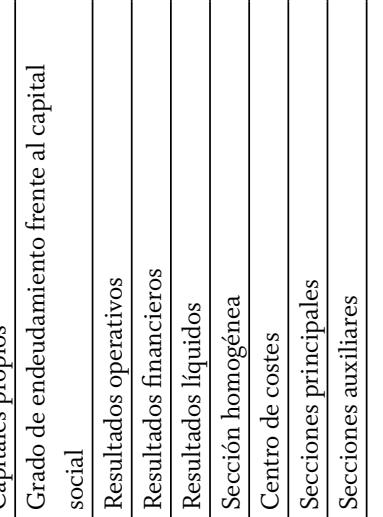

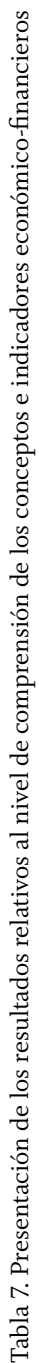


proceso de decisión de los profesionales de la salud. Estos profesionales, en particular los médicos, son un personal altamente cualificado y especializado, integrado en clases profesionales con fuerte tradición de autonomía profesional, habituados a practicar una medicina centrada en el paciente y no en los recursos. Sin embargo, las decisiones de estos profesionales, en especial las de los médicos, directa o indirectamente, determinan la cantidad y el carácter de los recursos consumidos. Esta constatación va en contra de una realidad en la cual los principios de carácter clínico compiten con los de carácter económico ("una vida no tiene precio pero alguien tiene que pagar su coste"), lo que hace inevitable la integración de la información médica con la de carácter económico; hay una presión creciente sobre el profesional clínico para reducir costos y al mismo tiempo mejorar la calidad (Pettersen \& Nyland, 2010). Sin embargo, la literatura evidencia la existencia de una cierta tensión en la relación entre los profesionales de la salud y los gestores profesionales en el hospital-empresa (Abernethy \& Stoelwinder, 1990; Armstrong, 2002; Cohn, Gill \& Schwartz, 2005; Mintzberg, 1979; Vlastarakos \& Nikolopoulos, 2008). Los profesionales están entrenados y socializados de acuerdo con modelos profesionales de control y desarrollan una fuerte orientación a los valores profesionales y normas que se consideran la antítesis de una orientación gerencial (Comerford \& Abernethy, 1999).

Reconocemos que los procesos de reforma del sistema de salud tienen una curva de aprendizaje necesariamente larga y constatamos, tal como señalan Richard B. Saltman, Josep Figueras y Constantino Sakellarides (1998), nor- malmente son necesarios varios años desde la implementación de una iniciativa antes de poder discernir cuál ha sido su impacto. Sin embargo, cualquier estudio que pueda lanzar algo de luz sobre la cuestión y contribuir a que administradores y políticos decidan con mayor información merece nuestro esfuerzo. Con este espíritu nos hemos arriesgado a realizar este proyecto de investigación, teniendo como base la idea de que no hay una teoría universal del cambio, ya que todas las prácticas sociales son inevitable e irremediablemente contextuales (Lawrence \& Doolin, 1997).

Aunque nuestro estudio es limitado en el contexto, entendemos que hace una contribución positiva de cara al esclarecimiento de las cuestiones de investigación:

1. ¿Los profesionales que tienen poder de decisión sobre la disposición de los recursos dentro del hospital-empresa, en particular los médicos y los cargos de jefe (director clínico, enfermero director y administradores), utilizan la información económicofinanciera disponible? Para responder a la primera cuestión se realizó un análisis de las funciones de los diversos grupos profesionales y se concluyó que, en el ámbito hospitalario, los profesionales que tienen poder de decisión sobre la utilización de los recursos son, esencialmente, los médicos y los cargos de jefe (director clínico, enfermeros directores y administradores). En el caso en estudio apenas el $65 \%$ de estos profesionales afirmaba consultar los documentos contables. Cuando se analizan los grupos de profesionales se constata que el $72 \%$ de los que ejercen car- 
gos de jefe consulta los documentos, frente a una tasa del 50\% de los médicos que no ejerce cargos de jefe. No obstante, el test del chi-cuadrado, realizado con el objetivo de determinar la asociación entre el cargo de jefe y el uso de información contable, no reveló la existencia de una asociación estadísticamente significativa entre las variables. En todo caso, los datos encontrados van en el mismo camino que los obtenidos en otros estudios internacionales, que revelan un bajo nivel de consulta de la información económico-financiera por los profesionales de la salud.

2. ¿Cuáles son los factores que condicionan la utilización de la información económicofinanciera?

Al intentar explorar los factores que condicionan la utilización de la información económicofinanciera, se constata que solo para las variables aceptación de la publicación de los resultados de la evaluación externa y aceptación del financiamiento del hospital en función de la producción, el grupo de usuarios de la información contable presenta diferencias estadísticamente significativas frente al grupo de los no usuarios. Los primeros tienen mayores niveles de concordancia con los dos principios fundamentales subyacentes a la nueva gestión pública, que enmarcan la empresarialización de los hospitales: la publicación de la evaluación externa y el financiamiento en función de la producción. Estos resultados sugieren que tal vez sea necesario informar mejor a los profesionales de salud sobre los procesos de reforma en curso.

Para los restantes factores potencialmente explicativos, no se revelan diferencias significati- vas; en el caso de las variables apreciación de los efectos económico-financieros de la empresarialización en el HE y nivel de comprensión de los conceptos e indicadores económico-financieros, podemos hablar de la existencia de tendencias. En el primer caso, el reconocimiento de los beneficios potenciales de la empresarialización del HE puede estar en el origen de una tendencia de mayor apertura a una nueva cultura empresarial. En el segundo caso, nos extrañamos de los resultados obtenidos, pues se esperaba una clara diferencia entre los dos grupos, dado su nivel de conocimiento de los indicadores económicofinancieros presentados en el cuestionario. Por eso, nos arriesgamos a avanzar con una explicación para aquello que consideramos una distorsión de la realidad: juzgamos que los sujetos que consultan los documentos tienen criterios de evaluación de su nivel de conocimiento de los indicadores económico-financieros más exigentes que aquellos que no los consultan. Los que consultan los documentos se enfrentan frecuentemente con sus limitaciones en el dominio de los conceptos, teniendo por eso una base de evaluación más concreta y sólida que aquellos que no los consultan. Esta cuestión merece, por tanto, un estudio más profundo con otro tipo de instrumento.

En relación con los indicadores clínicos, se destaca el bajo nivel de conocimiento del significado del índice de casemix que, siendo uno de los factores ponderados en el esquema de financiamiento del Hospital, revela el alejamiento de los profesionales de una cuestión absolutamente central en el rendimiento-desempeño económico-financiero de la institución. Esta constatación se refuerza por los resultados 
obtenidos con respecto al índice global de eficiencia hospitalaria.

3. ¿Con qué finalidad se utiliza la información económico-financiera?

Los documentos consultados más frecuentemente son la Memoria de actividades de los Hospitales S.A. (27,60\%) y el cuadro de mando integral del HE (30\%), precisamente aquellos que fueron introducidos en el proceso de empresarialización de los Hospitales. Por el contrario, los documentos menos consultados son el plan de negocios del HE y el contrato entre el HE y el Ministerio de Salud. Se trata de documentos internos fundamentales para la gestión del hospital, asociados con alguna confidencialidad y, por tanto, exigen un motivo y una motivación más explícita para su consulta.

En cuanto a la finalidad de la consulta, la más referenciada, en especial en lo que concierne a los documentos Memoria de actividades de los Hospitales S.A. y el Presupuesto de Servicio, fue la de apoyar una decisión en el ámbito de las funciones de los sujetos. En cuanto al Cuadro de Mando Integral, documento en relación con el cual se esperaba una elevada tasa de consulta con la finalidad de apoyar la toma de decisiones, el motivo más indicado fue la evaluación del desempeño de la administración del hospital. No obstante lo esperado, los datos obtenidos están en perfecta sintonía con los objetivos subyacentes al Cuadro de mando integral, ya que se trata del principal documento de base en el proceso de benchmarking entre los Hospitales S.A.

4. En el caso en que no utilizan la información económico-financiera, ¿cuál es el motivo?
Con respecto a las causas de la no consulta, nuestro estudio reveló que el principal motivo apuntado es el hecho de que los documentos no están disponibles. Estos resultados, ahora perceptibles debido a la confidencialidad de los datos, contrastan con el discurso del presidente del Consejo de Administración, que revelaba seguir una filosofía de comunicación y compartir la información. Además, este motivo es a veces indicado en relación con documentos de acceso público, lo que revela el desconocimiento de los sujetos sobre la publicidad de la información. En todo caso, la cuestión de la disponibilidad se mantiene, ya que se espera que la información se distribuya espontáneamente a quien la necesite y no que los sujetos la busquen deliberadamente.

Teniendo en cuenta los resultados obtenidos, en nuestros futuros proyectos de investigación sobre este tema, vamos a tratar de utilizar escalas ya probadas y basar nuestras hipótesis de estudio en marcos teóricos más enfocados.

\section{Referencias}

Abernethy, Margaret \& Stoelwinder, Johannes (1990). The Relationship between Organization Structure and Management Control in Hospitals: An Elaboration and Test of Mintzberg's Professional Bureaucracy Model. Accounting, Auditing \& Accountability Journal, 3 (3), 18-33. Abernethy, Margaret \& Vagnoni, Emidia (2004). Power, Organization Design and Managerial Behaviour. Accounting, Organizations and Society, 29 (3-4), 207-225. 
Ahrens, Thomas \& Dent, Jeremy F. (1998).

Accounting and Organizations: Realizing the Richness of Field Research. Journal of Management Accounting Research, 10, 1-39.

Aidemark, Lars-Göran (2001). Managed Health Care Perspectives: A Study of Management Accounting Reforms on Managing Financial Difficulties in a Health Care Organization. European Accounting Review, 10 (3), 545-560.

Armstrong, David (2002). Clinical Autonomy, Individual and Collective: the Problem of Changing Doctors' Behaviour. Social Science \& Medicine, 55, 1771-1777. Disponible en: http://www.academia.edu/458042/ Clinical_autonomy_individual_and_ collective_the_problem_of_changing_ doctors_behaviour

Arnaboldi, Michela \& Lapsley, Irvine (2004).

Modern Costing Innovations and Legitimation: A Health Care Study. Abacus, 40 (1), 1-20.

Brickley, James A.; Horn, R. Lawrence van \& Wedig, Gerard J. (2010). Board Composition and Nonprofit Conduct: Evidence from Hospitals. Journal of Economic Behavior \& Organization, 76 (2), 196-208.

Chua, Wai Fong (1986). Radical Developments in Accounting Thought. The Accounting Review, 61, 601-632.

Chua, Wai Fong (1995). Experts, Networks and Inscriptions in the Fabrication of Accounting Images: A Story of the Representation of Three Public Hospitals. Accounting, Organizations and Society, 20 (23), 111-145.
Chua, Wai Fong \& Preston, Alistair (1994). Worrying about Accounting in Health Care. Accounting, Auditing \& Accountability Journal, 7 (3), 4-17.

Cohn, Kenneth; Gill, Sandra L. \& Schwartz, Richard W. (2005). Gaining Hospital Administrators' Attention: Ways to Improve Physician - Hospital Management Dialogue. Surgery, 137 (2), 132-140.

Comerford, Sue E. \& Abernethy, Margaret (1999). Budgeting and the Management of Role Conflict in Hospitals. Behavioral Research in Accounting, 11, 93-110.

Drury, Colin \& Tayles, Mike (1995). Issues

Arising from Surveys of Management Accounting Practice. Management Accounting Research, 6 (3), 267-280.

Edwards, K. A. \& Emmanuel, Clive R. (1990).

Diverging Views on the Boundaries of Management Accounting. Management Accounting Research, 2 (1), 41-54.

Farnham, David \& Horton, Sylvia (1996). Managing Private and Public Organisations. En David Farnham \& Sylvia Horton (eds.). Managing the New Public Services, 25-46. London: MacMillan Press Ltd.

Gouveia, Miguel; Alvim, José Luís; Neto de Carvalho, Cristina; Meneses-Correia, José António \& Mota-Pinto, Manuela (2006). Resultados da Avaliação dos Hospitais SA. Lisboa: Comissão para Avaliação dos Hospitais Sociedade Anónima, CAHSA, Ministério de Saúde. Disponible en: http:// www.hospitaisepe.min-saude.pt/NR/ rdonlyres/AC1A014D-5D80-449F-8732DBF490284382/3721/Relatorio_CAHSA. pdf 
Grafton, Jennifer; Abernethy, Margaret A.

\& Lillis, Anne M. (2011). Organisational

Design Choices in Response to Public

Sector Reforms: A Case Study of Mandated

Hospital Networks. Management Accounting

Research, 22 (4), 242-268.

Hood, Christopher (1991). A Public

Management for All Seasons? Public

Administration, 69 (1), 3-19.

Hood, Christopher (1995). The New Public

Management in the 1980s: Variations on

a Theme. Accounting, Organizations and

Society, 20 (2/3), 93-109.

Hopwood, Anthony G. (1984). Accounting and

the Pursuit of Efficiency. En Anthony G.

Hopwood \& Cyril Tomkins (eds.). Issues in

Public Sector Management, 167-187. Oxford:

Philip Allan.

Kaplan, Robert (1983). Research Opportunities

in Management Accounting. Journal

of Management Accounting Research, 5,

1-14. Disponible en: http://staff.ui.ac.id/

system/files/users/ratnadewi_aaa/material/

kaplan1993.pdf

Keating, Patrick J. (1995). A Framework for

Classifying and Evaluating the Theoretical

Contributions of Case Research in

Management Accounting. Journal of

Management Accounting Research, 7, 66-86.

Kurunmäki, Liisa (2004). A Hybrid Profession -

the Acquisition of Management Accounting

Expertise by Medical Professionals.

Accounting, Organizations and Society, 29 (3-

4), 327-347.

Lapsley, Irvine (2001). The Changing Public

Sector: from Transition to Transformation.

European Accounting Review, 10 (3), 501-504.
Lawrence, Stewart; Alam, Manzurul \& Lowe,

Tony (1994). The Great Experiment:

Financial Management Reform in the New

Zealand Health Sector. Accounting, Auditing \& Accountability Journal, 7 (3), 68-95.

Lawrence, Stewart; Alam, Manzurul;

Northcott, Deryl \& Lowe, Tony (1997).

Accounting Systems as Systems of

Accountability in the New Zealand Health

Sector. Accounting, Auditing \& Accountability, 10 (5), 665-683.

Lawrence, Stewart \& Doolin, Bill (1997).

Introducing System Contradiction to Effect Change in the Public Sector: A New Zealand Case Study. International Journal of Public Sector Management, 10 (7), 490-504. Minogue, Martin (1998). Changing the State:

Concepts and Practice in the Reform of the Public Sector. En Martin Minogue, Charles Polidano \& David Hulme (eds.). Beyond the New Public Management: Changing Ideas and Practice in Governance, 17-37. Cheltenham: Edward Elgar Publishing Ltd.

Mintzberg, Henry (1979). The Structuring

of Organizations. Englewood Cliffs, New

Jersey: Prentice-Hall.

Pettersen, Inger Johanne (2001). Implementing Management Accounting Reforms in the Public Sector: the Difficult Journey from Intention to Effects. European Accounting Review, 10 (3), 561-581.

Pettersen, Inger Johanne \& Nyland, Kari (2010). Management and Control of Public Hospitals - The Use of Performance Measures in Norwegian Hospitals. A Case-Study. International Journal of Health Planning and Management, 21 (2), 133-149. 
Saltman, Richard B.; Figueras, Josep \&

Sakellarides, Constantino (1998). Critical

Challenges for Health Care Reform in Europe.

Buckingham, Philadelphia: World Health

Organization, Open University Press.

Scapens, Robert W. (1994). Never Mind the

Gap: towards an Institutional Perspective on Management Accounting Practice.

Management Accounting Research, 5 (3-4), 301-321.

Scarparo, Simona (2004). The Integration of

Clinical Governance, Medical Audit and Costing Information: Clinicians' Reinvention versus Managerial Assimilation. Working paper. University of Warwick.

Vlastarakos, Petros V. \& Nikolopoulos, Thomas P. (2008). The Interdisciplinary Model of Hospital Administration: Do Health Professionals and Managers Look at it in the
Same Way? European Journal of Public Health, 18 (1), 71-76.

Yin, Robert K. (1994). Case Study Research:

Design and Methods. London: Sage

Publications.

- Fecha de recepción: 30 de octubre de 2013

- Fecha de aceptación: 28 de enero de 2014

- Disponible en línea: 01 de julio de 2014

\section{Para citar este artículo}

Silva, Amélia Cristina Ferreira da \& Pereira, José Manuel Teixeira (2014). La perspectiva de los profesionales de la salud acerca de la información contable en un hospitalempresa en Portugal. Estudio de caso. Cuadernos de Contabilidad, 15 (38), 501-523.

doi: 10.11144/Javeriana.cc15-38.ppsa 
